\title{
Enhanced HLA typing performance of Korea Biobank Array with large scale reference panel
}

\author{
Sung Min Kim', Jong Cheol Jeong ${ }^{2}$, Dong Jin $\mathrm{Joo}^{3}$, Jaeseok Yang ${ }^{4}$, Myoung Soo Kim ${ }^{3}$, Hye-Mi Jang ${ }^{1}$, Hyun-Young Park ${ }^{5}$, \\ Bong-Jo Kim ${ }^{1}$ Young Jin Kim ${ }^{1}$
}

\footnotetext{
${ }^{1}$ Division of Genome Science, National Institute of Health, Cheongju, Korea

${ }^{2}$ Department of Internal Medicine, Seoul National University Bundang Hospital, Seongnam, Korea

${ }^{3}$ Department of Surgery, Yonsei University College of Medicine, Seoul, Korea

${ }^{4}$ Department of Surgery, Seoul National University Hospital, Seoul, Korea

${ }^{5}$ Department of Precision Medicine, National Institute of Health, Osong, Korea
}

Background: In clinical transplantation, increased human leucocyte antigen (HLA) mismatch between donor and recipient were well-known risk factor for graft survival. However, HLA typing in a large scale genome study is not feasible due to high cost. Alternatively, HLA alleles can be statistically inferred via HLA imputation from single nucleotide polymorphism (SNP) microarray data. Previously, Korea National Institute of Health $(\mathrm{KNIH})$ designed Korea Biobank Array (KBA), a fully customized SNP microarray for Koreans with 10K HLA regional SNPs as optimal set for imputing HLA alleles.

Methods: We assessed HLA typing accuracy of KBA comparing HLA imputed alleles from KBA and HLA typing via high-throughput sequencing. Among Korean Organ Transplantation Registry participants, 254 samples were genotyped using KBA and also HLA typed by HLAaccuTest kit (NGeneBio). For HLA imputation, IMPUTE v4 was used with reference panels of Pan-Asian \& Korean (PanREF, $\mathrm{n}=854$ ) or Han Chinese MHC (HanREF, $\mathrm{n}=10,689)$. Accuracy was assessed by comparing four-digit HLA alleles of the imputed and the NGS based typing among HLA-A, HLA-B, HLA-C, HLA-DRB1, HLA-DPA1, HPA-DPB1, HLA-DQA1, and HLADQB1 loci.

Results: Imputed HLA alleles were compared its true HLA alleles from NGS typing. For four-digit HLA alleles, accuracy was 88.9\% and $93.6 \%$ for PanREF and HanREF, respectively. While accuracy for HLA-DPA1 and HLA-DPB1 was comparable (95\%-96\%), HLA-C and HLA-DQA1 showed large difference in accuracy (51.7\%-91.5\% for PanREF, 82.2\%-99.1\% for HanREF). However, both panels showed comparable accuracy (about 95\%) for HLA-A, HLA-B, and HLA-DRB1, which are the most important three loci for transplantation. Overall, imputed HLA alleles from KBA using HanREF showed enhanced accuracy over PanREF.

Conclusions: Taken together, accuracy for HLA typing of KBA was increased using large reference panel (HanREF). The result showed that KBA is an efficient platform to comprehensively obtain four-digit level imputed HLA alleles with accuracy of about $94 \%$.

Corresponding author: Young Jin Kim

E-mail: anwltlarkr@gmail.com

(c) The Korean Society for Transplantation

This is an Open Access article distributed under the terms of the Creative Commons Attribution Non-Commercial License (http://creativecommons.org/licenses/by-nc/4.0/) which permits unrestricted non-commercial use, distribution, and reproduction in any medium, provided the original work is properly cited. 